\title{
EFFECT OF LOVASTATIN NANO DRUG DELIVERY SYSTEM ON BONE MINERAL DENSITY (BMD) AND BIOMECHANICAL PROPERTIES OF TIBIA BONES OF WISTAR RATS
}

\author{
RAMANDEEP KAUR*, MAKULA AJITHA
}

Centre for Pharmaceutical Sciences, Institute of Science and Technology, Jawaharlal Nehru Technological University Hyderabad (JNTUH), Hyderabad, Telangana State, India

Email: rkmann_87@yahoo.co.in

Received: 20 Jun 2019 Revised and Accepted: 30 Jul 2019

\section{ABSTRACT}

Objective: In the present study, transdermal nanoemulsion (NE) gel of lovastatin was investigated for its anti-osteoporosis effect on the long bones of rat i.e. tibia.

Methods: Male wistar rats ( $\mathrm{n}=30$, weighing 180-200g) were taken for this study and grouped as: 1) control (normal saline daily), 2) Dex (dexamethasone sodium; $25 \mathrm{mg} / \mathrm{kg}$ subcutaneously twice a week), 3) Dex+LNG5 (lovastatin nanoemulsion gel; $5 \mathrm{mg} / \mathrm{kg} / \mathrm{d}$ transdermally daily), 4) Dex+LNG10 (lovastatin nanoemulsion gel; $10 \mathrm{mg} / \mathrm{kg} / \mathrm{d}$ transdermally daily), and 5) Dex+ALN (alendronate sodium; $0.03 \mathrm{mg} / \mathrm{kg} / \mathrm{d}$ orally daily). All the treatments were carried out for $60 \mathrm{~d}$. At the end of the experiment, all animals were anesthetized using diethyl ether and collected blood samples from retro-orbital venous plexus of rats in dry eppendorf tubes followed by the sacrifice of animals by cervical dislocation method and collected tibia bones of both the legs for analysis.

Results: Bone formation biomarkers (OC: osteocalcin, b-ALP: bone-specific alkaline phosphatase, PINP: N-terminal propeptides of type I procollagen) were significantly improved and resorption biomarkers (CTx: C-terminal cross-linking telopeptides of type-I collagen, TRAcP5b: isoform 5b of tartarate resistant acid phosphatase) were significantly reduced in the LNG5 $(\mathrm{p}<0.05)$ and LNG10 $(\mathrm{p}<0.05)$ treatment groups when compared to Dex. In vivo antiosteoporotic results demonstrated the formation of new bone in osteoporotic rat tibias. Biomechanical strength testing demonstrated increased loadbearing capacity of rat tibias in the treated animals in comparison with the osteoporotic group ( $\mathrm{p}<0.05$ for LNG5 and $\mathrm{p}<0.01$ for LNG10).

Conclusion: Thus, the transdermal NE gel formulation of lovastatin demonstrated the greater potential for the treatment of osteoporosis.

Keywords: Nanoemulsion gel, Lovastatin, Bone biomarkers, Micro-CT, Bone mineral density, Biomechanical strength testing

(C) 2019 The Authors. Published by Innovare Academic Sciences Pvt Ltd. This is an open-access article under the CC BY license (http://creativecommons.org/licenses/by/4.0/) DOI: http://dx.doi.org/10.22159/ijpps.2019v11i9.34624

\section{INTRODUCTION}

Osteoporosis is a highly prevalent metabolic bone disorder characterized by low bone mineral density and deteriorated microarchitecture of bone [1]. It often results in hip, distal arm and spine fractures. The increased risk of fragility fractures in osteoporosis poses a threat to life expectancy and quality of life in both men and women. With advancing age, bone resorption increases in comparison to bone formation, which causes deterioration of bone and thus leads to osteoporosis. Osteoporosis is of two types, primary and secondary. Glucocorticoid (GC) induced osteoporosis is the most common form of secondary osteoporosis [2]. GC induces apoptosis of both osteoblasts and osteoclasts but increases the resorption process by increasing the life span of pre-existing osteoclasts [3].

There are various mechanisms by which GCs induce osteoporosis viz they suppress osteoblasts function which reduces bone remodeling and impairs bone tissue repair [4], inhibits the production of bone matrix components, reduces intestinal calcium absorption and increases renal calcium excretion leading to negative calcium balance [5]. GC's inhibit the osteoanabolic action of sex steroids and causes secondary hyperparathyroidism [6]. Food and drug administration (FDA) has approved various drugs for the treatment of osteoporosis viz. bisphosphonates (alendronate, risedronate, zoledronate) and recombinant parathyroid hormone [7, 8]. But all of them suffer from serious drawbacks. However, utilization of statins as anabolic agents [9, 10] in osteoporosis has spurred various experimental investigations.

Statins are the low molecular weight 3-hydroxy-3-methylglutaryl coenzyme A reductase inhibitor which shows the anabolic effect on osteoporotic bones through various mechanisms viz. promoting osteogenesis, suppressing apoptosis of osteoblasts and inhibiting osteoclastogenesis $[11,12]$. Statins suffer from the major limitation of the first-pass metabolism in the liver, which reduces their systemic availability to $2-4 \%$ approximately. The low concentrations of statins are not sufficient to reach bone tissue and produce osteogenic effects [13]. Thus local drug delivery systems need to be investigated to increase the concentration of statins reaching bone effectively. There are the number of approaches utilised by various researchers viz. multiple injections at single site $[14,15]$, implants $[16,17]$, scaffolds $[18,19]$, hydrogels $[20,21]$ etc. These approaches have provided significant results in improving osteoporosis but their major limitation is being invasive. Patient compliance is not taken care in these approaches. Transdermal nanoemulsion gel has shown quite significant improvement in osteoporosis [22, 23] and is the completely non-invasive approach.

In the present study; effect of transdermal nanoemulsion gel was studied. The aim of this investigation was to study the antiosteoporotic effect of transdermal lovastatin NE gel in the long bones i.e. tibia using glucocorticoid-induced osteoporosis rat model.

\section{MATERIALS AND METHODS}

\section{Chemicals and reagents}

Lovastatin was obtained as a gift sample from Panacea Biotec, New Delhi. Labrafac PG and transcutol P were obtained as a gift sample from Gattefosse. Tween 80, diethyl ether, isopropyl alcohol, triethanolamine, phosphoric acid, sodium chloride and carbopol 940 was procured from SD fine chemicals. Water was obtained from Milli-Q water purification system. Biochemical kits specific for alkaline phosphatase (ALP), calcium, phosphorus, total protein (TP), serum glutamate oxaloacetate transaminase (SGOT), serum glutamate pyruvate transaminase (SGPT), triglyceride and total cholesterol were procured from Accurex, Mumbai, India. OC, b-ALP, PINP, CTx, TRAcP5b rat specific ELISA kits were procured from Shanghai YL Biotech Co. Ltd., China. 


\section{Experimental animals}

Thirty male albino wistar rats, three months of age and weighing approximately 180-200 g were procured from Sanzyme Ltd., Hyderabad. Animals were maintained under controlled conditions of $20{ }^{\circ} \mathrm{C}-25{ }^{\circ} \mathrm{C}$ temperature, $55-60 \%$ humidity, $12 \mathrm{~h}$ light/dark cycle and fed on standard chow diet and water ad libitum. Their weights were recorded both before and after end of the study. The study design was approved by the Institutional Animal Ethics Committee (009/MRIPS/CPCSEA-IAEC/Hyd/2016).

\section{Preparation of lovastatin loaded NE gel}

The NE was prepared using aqueous phase titration method. Firstly, lovastatin $(5 \mathrm{mg} / \mathrm{ml})$ was added to $5 \% \mathrm{v} / \mathrm{v}$ of labrafac PG and shaken continuously till a clear solution is obtained. To this solution, $30 \%$ $\mathrm{v} / \mathrm{v}$ of tween 80 and $30 \% \mathrm{v} / \mathrm{v}$ of transcutol was added. The mixture was vortexed for $1-2 \mathrm{~min}$. Then finally $35 \% \mathrm{v} / \mathrm{v}$ of water was added and the mixture was shaken vigorously to obtain a clear, homogenous and transparent mixture. This results in the preparation of lovastatin loaded NE. In order to prepare NE gel, carbopol 940 polymer $(1 \mathrm{~g})$ was firstly dissolved in $100 \mathrm{ml}$ of water and allowed to swell for $24 \mathrm{~h}$ in dark place. To the swelled polymer, $10 \mathrm{~g}$ each of propylene glycol and isopropyl alcohol were added and mixed continuously for 10-15 min., followed by addition of $0.5 \mathrm{~g}$ of triethanolamine which resulted in the formation of clear translucent gel $[14,24]$. Finally, lovastatin loaded NE was mixed with $1 \mathrm{~g}$ of above-prepared gel to result in the formation of lovastatin loaded $\mathrm{NE}$ gel (LNG5). Similarly, LNG10 was prepared wherein $10 \mathrm{mg} / \mathrm{ml}$ of lovastatin was added to $5 \% \mathrm{v} / \mathrm{v}$ of labrafac $\mathrm{PG}$ and prepared formulation henceforth.

\section{Experimental design}

One group of animals $(n=6)$ received subcutaneous injection of normal saline $(1 \mathrm{ml} / \mathrm{kg} / \mathrm{d})$ for $60 \mathrm{~d}$. This group served as control. The second group $(\mathrm{n}=24)$ received dexamethasone sodium (Dex) $(25 \mathrm{mg} / \mathrm{kg})$ subcutaneously twice a week for $60 \mathrm{~d}$ to induce osteoporosis. Blood samples were collected from both the group of animals after 30 and 60 $\mathrm{d}$ to analyse the levels of bone turnover markers and confirm osteoporosis induction. Treatments were started after induction of osteoporosis. The second group animals were further divided into treatment groups. The third group was administered lovastatin nanoemulsion gel (Dex+LNG5; $5 \mathrm{mg} / \mathrm{kg} / \mathrm{d}$ ) transdermally daily, fourth group received lovastatin nanoemulsion gel (Dex+LNG10; 10 $\mathrm{mg} / \mathrm{kg} / \mathrm{d}$ ) transdermally daily, and fifth group received alendronate sodium (Dex+ALN; $0.03 \mathrm{mg} / \mathrm{kg} / \mathrm{d}$ ) orally daily for $60 \mathrm{~d}$.

At the end of the experiment, all animals were anesthetized using diethyl ether and blood samples were collected from retro-orbital venous plexus of rats in dry eppendorf tubes. Blood samples were allowed to clot, centrifuged at $3000 \mathrm{rpm}$ for $15 \mathrm{~min}$ and serum separated was collected and stored at $-20^{\circ} \mathrm{C}$ until further analysis. Immediately after this, animals were sacrificed by cervical dislocation method and collected tibia bones of both the legs. Tibia bones were weighed, rinsed with phosphate buffer saline (PBS) and stored at $-20{ }^{\circ} \mathrm{C}$ after wrapping in gauze soaked with PBS until further investigation [25].

\section{Biochemical assay}

The serum samples stored at $-20{ }^{\circ} \mathrm{C}$ were thawed at room temperature first and analysed for albumin, alkaline phosphatase (ALP), calcium, cholesterol, creatinine, glucose, phosphorus, serum glutamate oxaloacetate transaminase (SGOT), serum glutamate pyruvate transaminase (SGPT), triglyceride and total protein. The samples were analyzed using fully automatic autoanalyzer (Accurex, Sphera autoanalyzer, Mumbai, India) [26].

\section{Bone turnover marker assay}

Stored serum samples were first thawed at room temperature and used for evaluation of various bone turnover markers such as bone formation markers (OC: osteocalcin, b-ALP: bone-specific alkaline phosphatase, PINP: N-terminal propeptides of type I procollagen) and bone resorption markers (CTx: C-terminal cross-linking telopeptides of type-I collagen, TRAcP5b: isoform $5 b$ of tartarate resistant acid phosphatase). These markers were analysed using commercially available rat specific ELISA kits [26].

\section{Micro-CT evaluation}

The stored tibia bones were thawed at room temperature before subjecting to Micro-CT analysis. During scanning, all tibia bones were wrapped in gauze soaked with phosphate buffer to prevent dehydration and scanned using X-radia versa XRM 500 Micro-CT scanner (X-radia, Zeiss, Germany). The radiographs were obtained longitudinally at proximal distal region of tibia. The parameters were set at $80 \mathrm{kV}$ voltage, $7.0 \mathrm{~W}$ power and $86.7 \mu \mathrm{A}$ current with an exposure time of $10 \mathrm{sec}$, and voxel resolution of $13 \mu \mathrm{m}$. Tomographic image reconstruction was performed using XM reconstructor and XM3D viewer. The proximaldistal cancellous bone microarchitecture was quantified for bone mineral density (BMD, $\mathrm{g} / \mathrm{cm}^{3}$ ), bone volume to tissue volume ratio (BV/TV, \%), trabecular bone thickness ( $\mathrm{TbTh}, \mathrm{mm}$ ), trabecular number (Tb. N, 1/mm) and trabecular separation (Tb. Sp, mm) [27].

\section{Biomechanical bone strength testing}

The biomechanical strength testing was performed on the compact bone using three-point bending test utilising $25 \mathrm{kN}$ Universal testing machine (BiSS Makron, Bangalore, India). All the bone samples were thawed at room temperature before testing. For the test, the length of the bone samples was measured using vernier calliper. Tibia was placed horizontally on two supporting bars located at a span distance of $14 \mathrm{~mm}$ and third bar carefully positioned perpendicularly at the mid-axis of tibia bone (fig. 1). Load was applied at a displacement rate of $1 \mathrm{~mm} / \mathrm{sec}$ until the bone fractures. The load-displacement curves were recorded (fig. 2) and calculated stiffness $(\mathrm{N} / \mathrm{mm})$, peak load $(\mathrm{N})$, ultimate strength $(\mathrm{mPa})$ and Young's modulus (GPa) from the curve $[28,29]$. All data were represented as mean \pm SD.
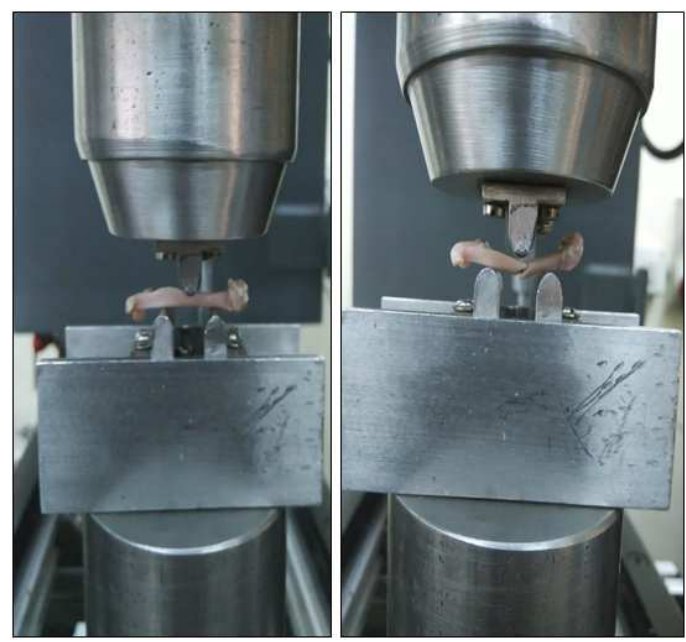

Fig. 1: Representation of three-point bending test.

\section{Statistical analysis}

Data is represented as mean $\pm S D$. For comparing different treatment groups, one-way analysis of variance (ANOVA) was utilized, followed by Tukey's multiple comparison test using Graph Pad Prism 5 software (version 5.0). $\mathrm{p}<0.05$ was considered significant.

\section{RESULTS}

\section{Biochemical assay}

Serum albumin, ALP, calcium, cholesterol, creatinine, glucose, phosphorus, SGOT, SGPT, triglyceride, and total protein levels are represented in table 1. After induction of osteoporosis, serum ALP levels were significantly elevated in comparison with the control rats. Induction of osteoporosis using dexamethasone reduced the calcium and phosphorus levels as indicated in table 1 and significant improvements were observed in their levels after $60 \mathrm{~d}$ of treatment with LNG5 (containing a low dose of lovastatin) and LNG10 (containing a high dose of lovastatin). The results were also comparable with the standard 


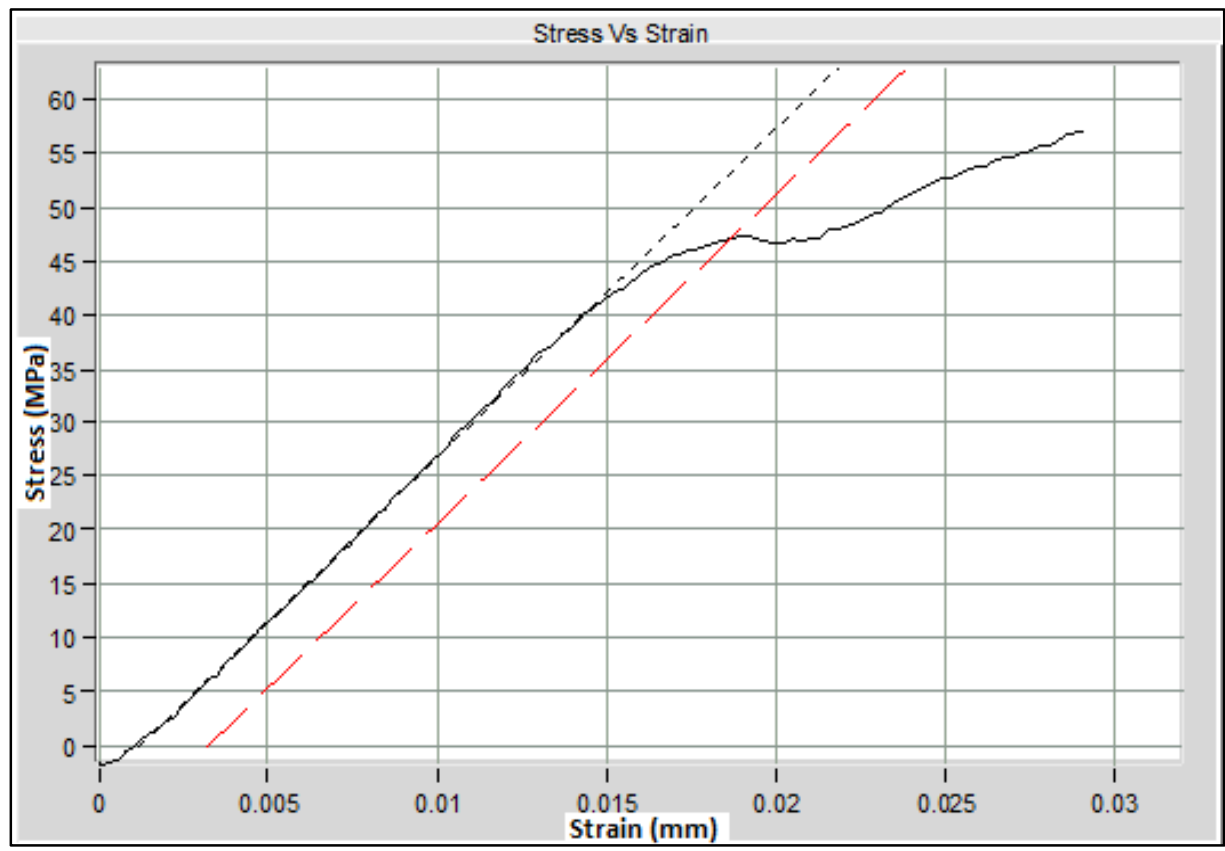

Fig. 2: Load (stress) displacement (strain) curve recorded from the three-point bending test.
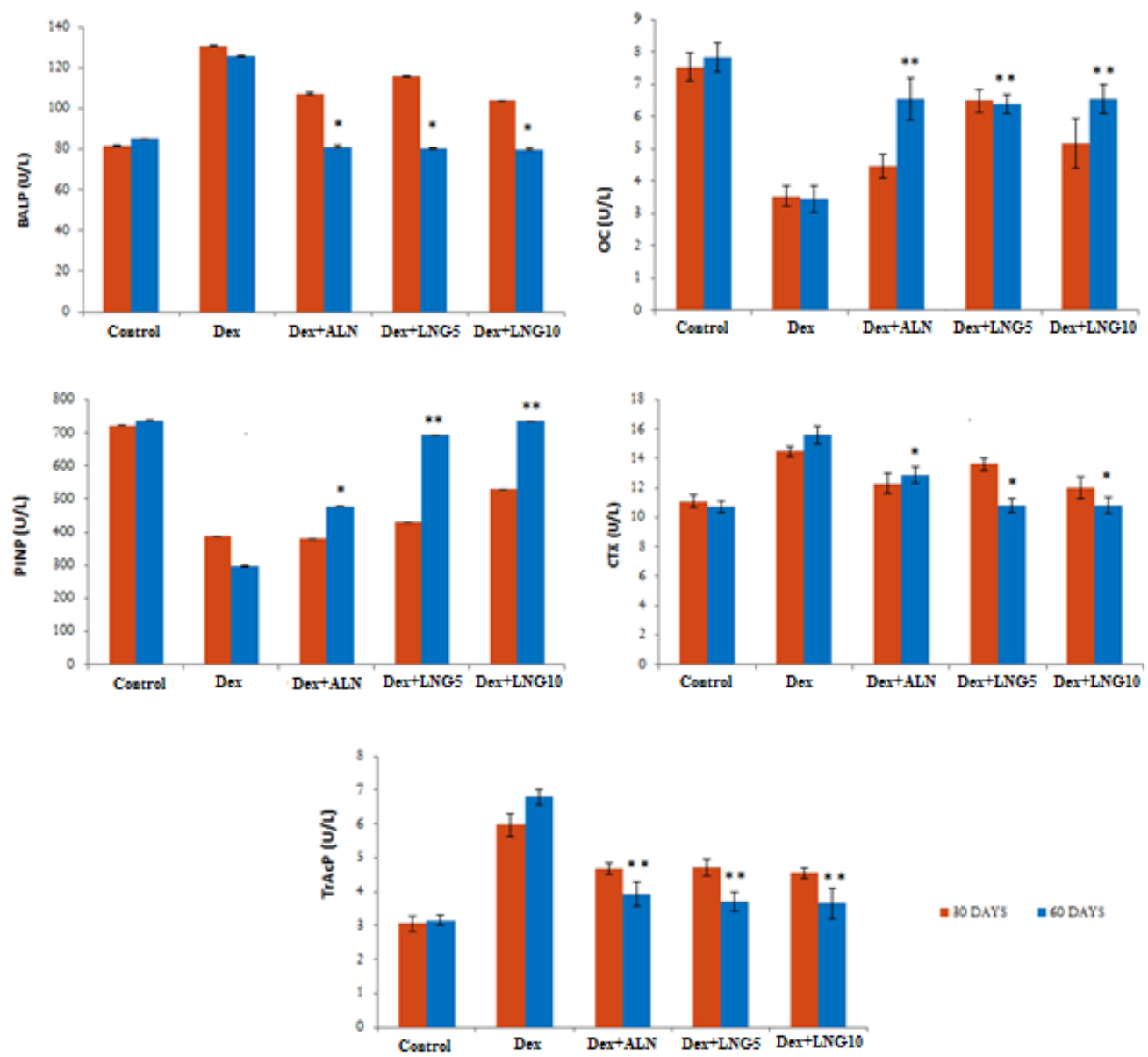

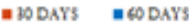

Fig. 3: Comparison of biomarker levels (a) BALP: bone-specific alkaline phosphatase, (b) OC: osteocalcin, (c) PINP: N-terminal propeptides of type I procollagen, (d) CTx: C-terminal cross-linking telopeptides of type-I collagen, (e) TRAcP5b: isoform $5 \mathrm{~b}$ of tartrate resistant acid phosphatase in rat blood serum of various treated groups. ${ }^{*} \mathbf{p}<0.05$, and ${ }^{* *} \mathbf{p}<0.01$ (ANOVA followed by Tukey's multiple comparison test). 
Table 1: Biochemical parameters analysed in blood sera of rats $(n=6)$

\begin{tabular}{lllll}
\hline & Control & Dex & Dex+LNG 5 & Dex+LNG 10 \\
\hline Alb (mg/dl) & $3.59 \pm 1.9$ & $2.35 \pm 0.98^{\mathrm{b}}$ & $2.97 \pm 1.23$ & $3.23 \pm 1.11$ \\
ALP (U/l) & $291.04 \pm 19.9$ & $485.1 \pm 20.1^{\mathrm{b}}$ & $374.2 \pm 14.1^{* *}$ & $328.6 \pm 17.2^{* *}$ \\
Ca (mg/dl) & $7.48 \pm 0.37$ & $6.067 \pm 0.42^{\mathrm{a}}$ & $6.773 \pm 0.89$ & $3.64 \pm 1.03$ \\
Chol (mg/dl) & $12.03 \pm 0.86$ & $7.017 \pm 0.87^{\mathrm{b}}$ & $9.523 \pm 0.53^{*}$ & $309.2 \pm 12.2^{* *}$ \\
Cr (mg/dl) & $0.616 \pm 0.02$ & $0.913 \pm 0.05$ & $0.765 \pm 0.03$ & $9.735 \pm 1.55^{*}$ \\
Glu (mg/dl) & $61.95 \pm 0.23$ & $50.42 \pm 0.41$ & $56.18 \pm 0.56$ & $0.725 \pm 0.03$ \\
P (mg/dl) & $9.56 \pm 0.69$ & $7.1 \pm 0.31^{\mathrm{b}}$ & $8.33 \pm 0.30^{*}$ & $59.78 \pm 0.82$ \\
SGOT (U/l) & $72.62 \pm 1.14$ & $110 \pm 2.35^{\mathrm{b}}$ & $91.31 \pm 2.40^{* *}$ & $8.67 \pm 0.55^{*}$ \\
SGPT (U/l) & $4.18 \pm 0.25$ & $11.5 \pm 0.33^{\mathrm{b}}$ & $7.873 \pm 0.48$ & $87.67 \pm 1.89^{* *}$ \\
Tg (mg/dl) & $27.36 \pm 0.86$ & $10.2 \pm 1.75^{\mathrm{b}}$ & $18.78 \pm 0.77^{* *}$ & $7.953 \pm 0.21$ \\
TP (mg/dl) & $4.48 \pm 0.11$ & $4.133 \pm 0.13$ & $4.307 \pm 0.25$ & $17.89 \pm 0.55^{* *}$ \\
\hline
\end{tabular}

Data are expressed as mean $\pm \mathrm{SD}, \mathrm{N}=6$ animals per group. Alb albumin, $A L P$ alkaline phosphatase, $C a$ calcium, $C h o l$ total cholesterol, $C r$ Creatinine, $G l u$ glucose, $P$ phosphorus, $S G O T$ serum glutamate oxaloacetate transaminase, SGPT serum glutamate pyruvate transaminase $T g$ triglyceride, $T P$ total protein. ${ }^{a} \mathrm{p}<0.05,{ }^{\mathrm{b}} \mathrm{p}<0.001$ when compared to control (ANOVA followed by Tukey's multiple comparison test). ${ }^{*} \mathrm{p}<0.05,{ }^{* *} \mathrm{p}<0.001$ when ${ }^{2}$ compared to Dex (ANOVA followed by Tukey's multiple comparison test).

\section{Bone turnover marker assay}

The levels of CTx, TrAcP and b-ALP were highly elevated after two months of treatment with dexamethasone indicating induction of osteoporosis, while the levels of OC and PINP were highly reduced (fig. 3). Significant changes were observed in the levels of BTMs after $60 \mathrm{~d}$ of treatment post osteoporosis induction. It was observed that b-ALP $(\mathrm{p}<0.05)$ levels reduced significantly and OC $(\mathrm{p}<0.05)$ and PINP $(p<0.05)$ levels increased significantly when compared with the Dex group after treatment with both LNG5 and LNG10. Meanwhile, CTx $(\mathrm{p}<0.05)$ and $\operatorname{TrAcP}(\mathrm{p}<0.05)$ levels were reduced significantly in the treated groups LNG5 and LNG10 when compared to Dex.

\section{Micro-CT evaluation}

Micro-CT radiographs obtained using X-radia versa XRM 500 Micro-CT scanner (X-radia, Zeiss, Germany) are represented in fig. 4. It clearly demonstrated reduction of trabecular integrity, lacunae and decreased thickening of the trabecular wall in Dex group after induction of osteoporosis in comparison to control group thus indicating that osteoporosis was established. This was also evident from the quantitative parameters analysed the wherein significant change in BV/TV, Tb. N, Tb. Th and Tb. Sp levels (fig. 5) was observed in Dex group in comparison to normal rats $(\mathrm{p}<0.001$ for all parameters).
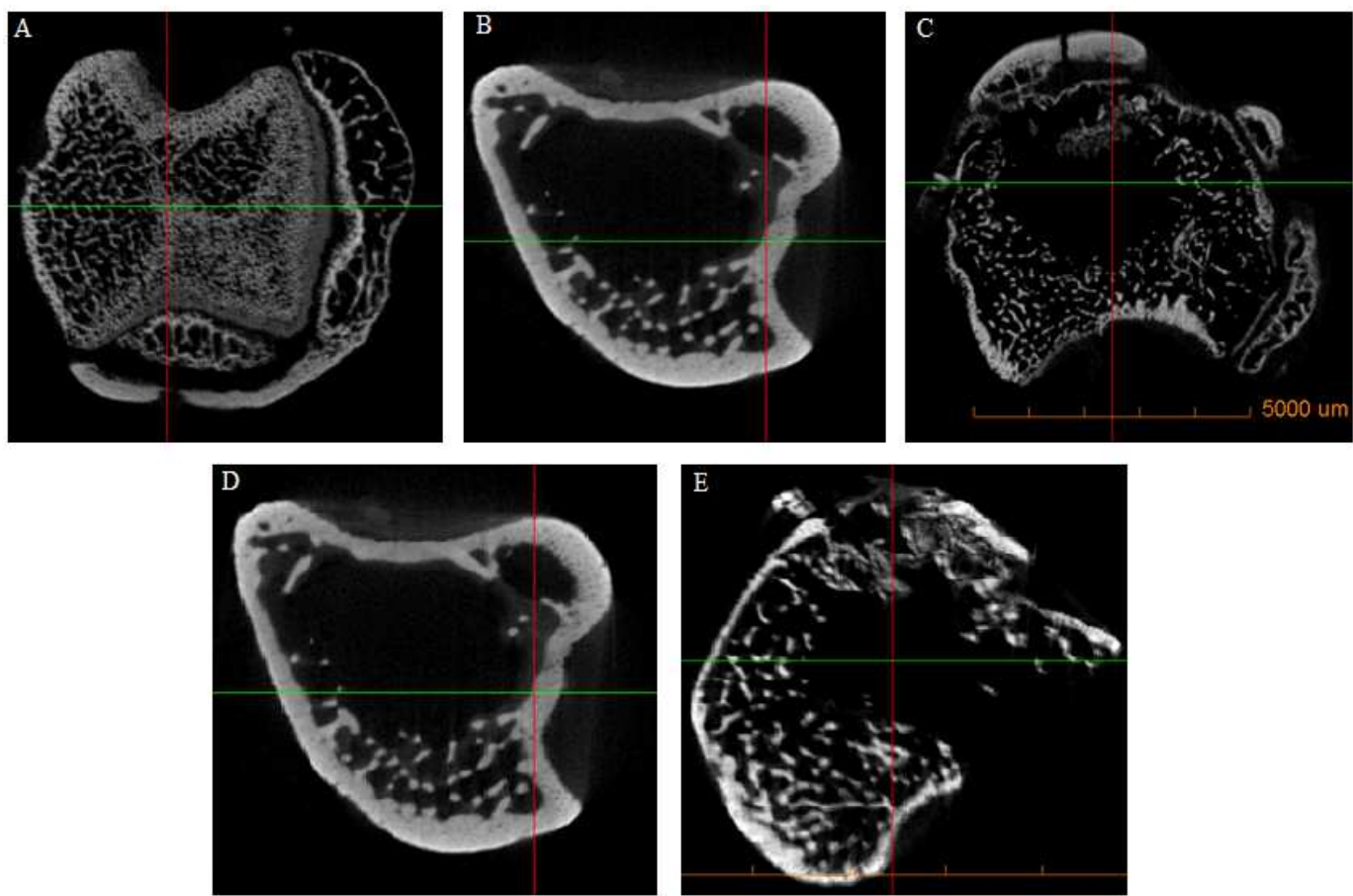

Fig. 4: Representative micro-CT images of distal femur head of rat in various treated groups. a) Control b) Dex: dexamethasone sodium, c) Dex+ALN: standard treatment alendronate, d) Dex+LNG5: lovastatin nanoemulsion gel $(5 \mathrm{mg} / \mathrm{ml})$, e) Dex+LNG10; lovastatin nanoemulsion gel $(10 \mathrm{mg} / \mathrm{ml})$

Micro-CT radiographs of the treatment groups (LNG5, LNG10 and ALN groups) showed an increase in trabecular integrity, reduction in lacunae and improvement in thickening of the trabecular wall. The trabecular bone parameters assessed also showed improvements in BV/TV, Tb. N, Tb. Th and Tb. Sp levels. BV/TV ( $<<0.01$ for ALN, LNG5 and LNG10), Tb. $\mathrm{N}(\mathrm{p}<0.05$ for ALN and LNG10), and Tb. Th levels were significantly increased $(\mathrm{p}<0.05)$ while $\mathrm{Tb}$. Sp was significantly reduced $(\mathrm{p}<0.01$ for ALN, LNG5 and LNG10), in comparison with Dex (fig. 5). This effect was demonstrated dose-dependently as LNG10 group showed more improvements in the BV/TV, Tb. N, Tb. Th and Tb. Sp levels than the LNG5 group. BMD also showed significant improvement in the treatment groups ( $\mathrm{p}<0.05$ for ALN, LNG5 and LNG10) in comparison to Dex. 

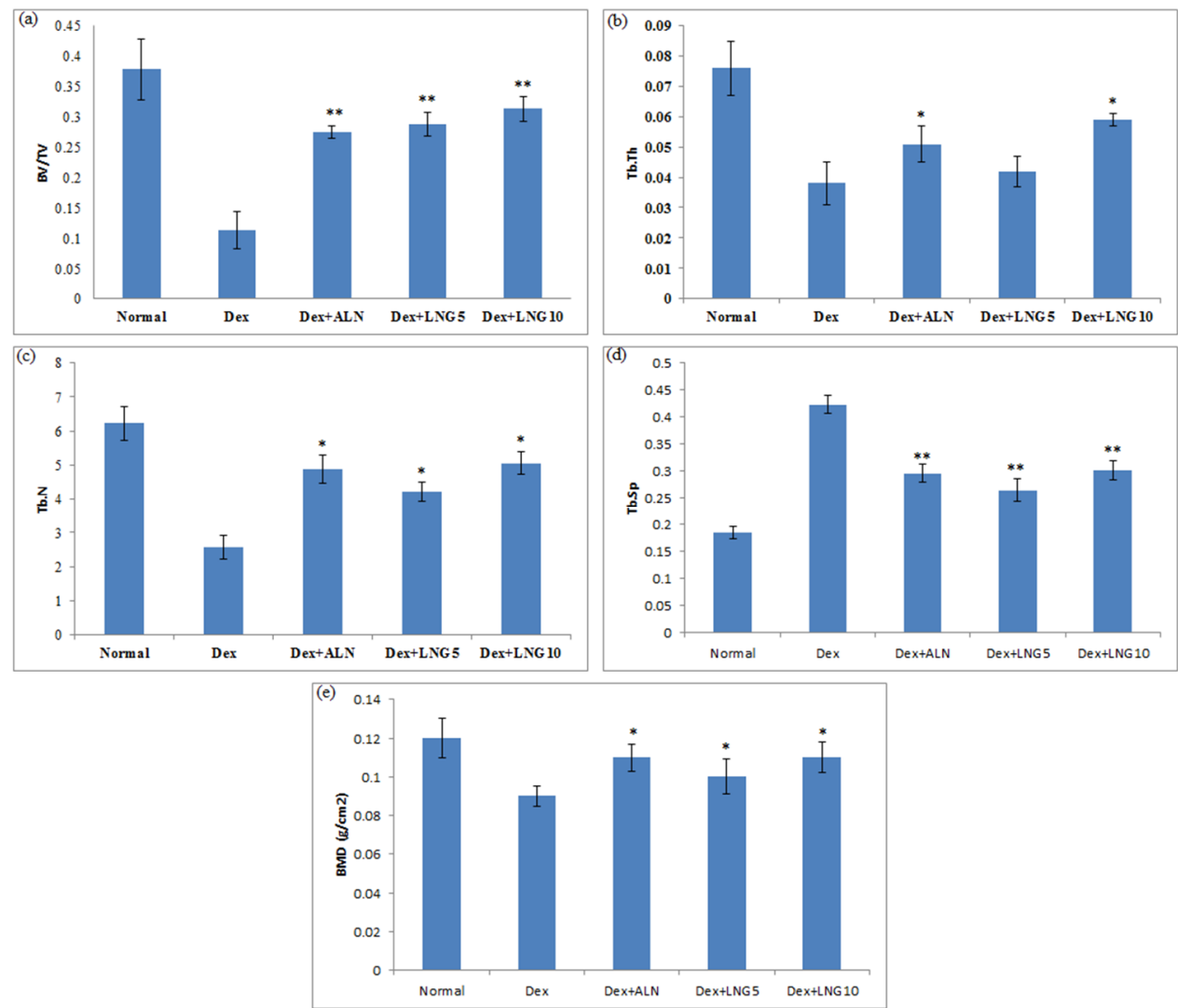

Fig. 5: Comparison of microstructural trabecular bone parameters measured at proximal tibia region of various groups, (a) BV/TV: bone volume to tissue volume ratio, (b) Tb. Th: trabecular thickness, (c) Tb. N: trabecular number, (d) Tb. Sp: trabecular separation, (e) BMD: bone mineral density. ${ }^{*} \mathbf{p}<0.05,{ }^{* *} \mathbf{p}<0.01$ versus Dex (calculated using ANOVA followed by Tukey's multiple comparison test)

\section{Biomechanical strength testing}

The extracted rat tibia bones were subjected to three-point bending test and measured peak load, ultimate strength, stiffness, and young's modulus of elasticity of the bones. After osteoporosis induction using dexamethasone, significant reductions in peak load $(\mathrm{p}<0.05)$, stiffness $(\mathrm{p}<0.05)$ and ultimate strength $(\mathrm{p}<0.05)$ was observed in the Dex group when compared to control (fig. 6). The treatment groups LNG5 and LNG10 showed significant improvements in peak load $(\mathrm{p}<0.05$ for LNG5 and $\mathrm{p}<0.01$ for LNG10), stiffness ( $p<0.05$ for LNG5 and $p<0.01$ for LNG10) and ultimate strength ( $\mathrm{p}<0.01$ for LNG10).
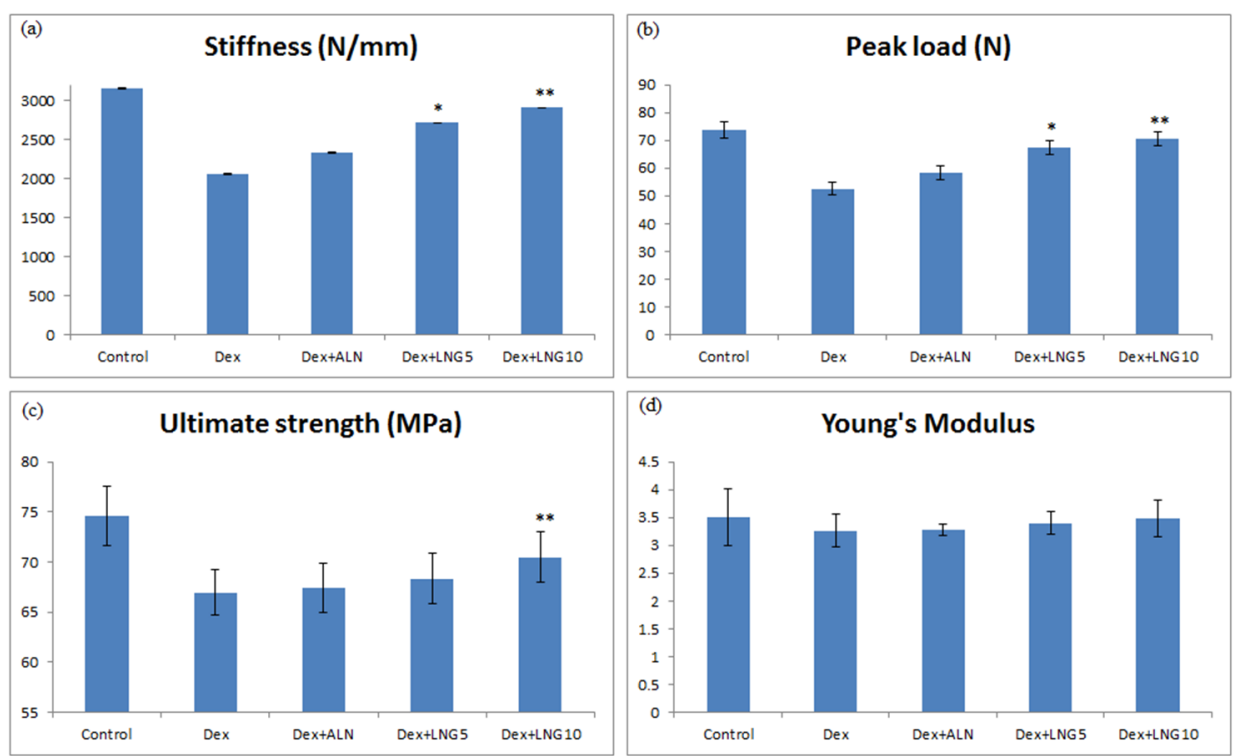

Fig. 6: Biomechanical strength testing of rat tibia representing (a) stiffness (N/mm), (b) peak load (N), (c) ultimate strength (MPa), and (d) youngs modulus of elasticity (GPa). Bars represent mean \pm SD of 6 animals per group, statistically analyzed using ANOVA followed by Tukey's multiple comparison test where ${ }^{*} \mathrm{p}<0.05$ and ${ }^{* *} \mathrm{p}<0.01$ versus Dex 


\section{DISCUSSION}

The analysis of various biochemical parameters in blood serum help in understanding the trend of biological processes. The serum ALP levels were significantly $(\mathrm{p}<0.001)$ elevated after dexamethasone treatment, thereby indicating the establishment of osteoporosis in the rats. This was further supported by a reduction in the serum calcium and phosphorus levels. Other parameters viz albumin creatinine, glucose, total protein, SGOT, and SGPT did not show any significant changes, thus ruling out the possibility of other effects.

Biochemical monitoring of bone metabolism i.e. resorption and formation can be carried out by analysing various enzymes and nonenzymatic peptides derived from the cellular and noncellular compartments of bone. These biomolecules released into blood circulation are termed as "Bone turnover markers" (BTM). BTMs released during different formation phases of active osteoblasts represent the bone formation markers such as b-ALP, OC and PINP While the BTMs released during bone resorption process carried by osteoclasts form the bone resorption markers such as CTx and TRACP. BTMs respond rapidly to the changes in bone physiology and thus are widely utilised for understanding changes in the bone during osteoporosis development and treatment [30]. Bone formation markers were significantly improved and bone resorption markers were significantly reduced after treatment with lovastatin NE gel formulations (LNG5 and LNG10) in comparison with Dex group. These improvements in the BTMs were almost similar to the alendronate treated group thus suggesting that prepared lovastatin $\mathrm{NE}$ gel preparations could potentially increase bone formation in the rats after osteoporosis induction with dexamethasone.

Micro-CT findings were quite significant in this research as they provide substantial data to support the objective of this research work. The radiographs showed an increase in trabecular integrity, reduction in lacunae, and improvement in thickening of the trabecular wall after treatment with NE gel in comparison with Dex group. The trabecular bone parameters BV/TV, Tb. N, Tb. Th and Tb. Sp levels of Dex+LNG5 and Dex+LNG10 groups were significantly improved in comparison with the Dex group, thus indicating the efficacy of prepared NE gel formulations of lovastatin. Moreover, the improvements were more significant in LNG10 group than LNG5 group, thus indicating a dose-dependent effect. Similar findings were reported by us in the femur bone as well [22].

The biomechanical strength testing was carried out to study the load-bearing capacity of the tibia bones before and after treatment with lovastatin NE gel. Dex+LNG5 and Dex+LNG10 groups showed improvement in peak load, ultimate stress, stiffness and young's modulus of elasticity in comparison with osteoporosis group (Dex), thus indicating an increase in the load-bearing capacity of the rat tibia. The significant improvement in biomechanical strength testing parameters indicates the effectiveness of the lovastatin NE gel formulation in osteoporosis.

\section{CONCLUSION}

Overall, the transdermal NE gel of lovastatin showed positive effects on the dexamethasone-induced osteoporotic rat tibia bones. Moreover, significant improvements were seen dose-dependently. Thus the present animal study suggests it could be an alternative therapy for osteoporosis, however further clinical studies should be intervened to explore its full potential.

\section{ACKNOWLEDGMENT}

The authors are thankful to the Department of Science and Technology (DST), Government of India, New Delhi, India for providing financial support through Women Scientist-A Fellowship (File. No. DST/WOS-A/2014/LS-1234).

\section{AUTHORS CONTRIBUTIONS}

Mrs Ramandeep Kaur carried out the experimental work, drafted, reviewed and finalized the manuscript. Dr Makula Ajitha supervised the work and edited the manuscript.

\section{CONFLICT OF INTEREST}

The authors declare no conflict of interest.

\section{REFERENCES}

1. Deal C. Potential new drug targets for osteoporosis. Nat Clin Pract Rheumatol 2009;5:20-7.

2. Sadat Ali M, Alelq AH, Alshafei BA, Al Turki HA, Abujubara MA Osteoporosis prophylaxis in patients receiving chronic glucocorticoid therapy. Ann Saudi Med 2009:29:215-8.

3. Migliaccio S, Brama M, Fornari R, Greco EA, Spera G, Malavolta $\mathrm{N}$. Glucocorticoidinduced osteoporosis: an osteoblastic disease. Aging Clin Exp Res 2007;19:5-10.

4. Lane NE, Yao W. Glucocorticoid-induced bone fragility. Ann N Y Acad Sci 2010;1192:81-3.

5. Ferrari P. Cortisol and the renal handling of electrolytes: role in glucocorticoid-induced hypertension and bone disease. Best Pract Res Clin Endocrinol Metab 2003;17:575-89.

6. Ahmeda HH, Morcosb NYS, Eskandera EF, Seoudib DMS, Shalbya AB. Role of dehydroepiandrosterone in management of glucocorticoid-induced secondary osteoporosis in female rats. Exp Toxicol Pathol 2012;64:659-64.

7. Honig S, Rajapakse CS, Chang G. Current treatment approaches to osteoporosis. Bull Hosp Jt Dis 2013;71:184-8.

8. Khajuria DK, Razdan R, Mahapatra DR. Drugs for the management of osteoporosis: a review. Rev Bras Reumatol 2011;51:365-82.

9. Mundy GR. Statins and their potential for osteoporosis. Bone 2001;29:495-7.

10. Mundy G, Garrett R, Harris S, Chan J, Chen D, Rossini G, et al. Stimulation of Bone Formation in vitro and in rodents by statins. Science 1999;286:1946-9.

11. Ruan F, Zheng Q, Wang J. Mechanisms of bone anabolism regulated by statins. Biosci Rep 2012;32:511-9.

12. Zhang Y, Bradley AD, Wang D, Reinhardt RA. Statins, bone metabolism and treatment of bone catabolic diseases. Pharmacol Res 2014;88:53-61.

13. Haberstadt C, Anderson P, Bartel R, Cohen R, Naughton G. Physiological cultured skin substitutes for wound healing. Mater Res Soc Symp Proc 1992;252:323-30.

14. Masuzaki T, Ayukawa Y, Moriyama Y, Jinno Y, Atsuta I, Ogino Y, et al. The effect of a single remote injection of statinimpregnated poly (lactic-co-glycolic acid) microspheres on osteogenesis around titanium implants in rat tibia. Biomaterials 2010;31:3327-34.

15. Zou Y, Brooks JL, Talwalkar V, Milbrandt TA, Puleo DA. Development of an injectable two-phase drug delivery system for sequential release of antiresorptive and osteogenic drugs. J Biomed Mater Res B Appl Biomater 2012;100:155-62.

16. Xiangning L, Xiaoran L, Shaobing L, Xiaosong Z, Sha L, Qiangbin W. An In vitro Study of a titanium surface modified by simvastatin-loaded titania nanotubes-micelles. J Biomed Nanotechnol 2014;10:194-204.

17. Stadlinger P, Korn N, Tödtmann U, Eckelt U, Range A, Bürki SJ, et al. Osseointegration of biochemically modified implants in an osteoporosis rodent model. Eur Cells Mater 2013;25:326-40.

18. Yoshii T, Hafeman AE, Nyman JS, Esparza JM, Shinomiya K, Spengler DM, et al. A sustained release of lovastatin from biodegradable, elastomeric polyurethane scaffolds for enhanced bone regeneration. Tissue Eng Part A 2010;16:236979.

19. Chou J, Ito T, Bishop D, Otsuka M, Ben-Nissan B. Controlled release of simvastatin from biomimetic b-TCP drug delivery system. PLoS ONE 2013;8:e54676

20. Park YS, David AE, Park KM, Lin C, Than KD, Lee K, et al. Controlled release of simvastatin from in situ forming hydrogel triggers bone formation in MC3T3-E1 cells. AAPS J 2013;15:367-76.

21. Tanabe $K$, Nomoto $H$, Okumori $N$, Miura T, Yoshinari $M$. Osteogenic effect of fluvastatin combined with biodegradable gelatin hydrogel. Dent Mater J 2012;31:489-93.

22. Kaur R, Ajitha M. Formulation of transdermal nanoemulsion gel drug delivery system of lovastatin and its in vivo 
characterization in glucocorticoid-induced osteoporosis rat model. J Drug Delivery Sci-Tech 2019;52:968-78.

23. Kaur R, Ajitha M. Transdermal delivery of fluvastatin loaded nanoemulsion gel: preparation, characterization and in vivo anti-osteoporosis activity. Eur J Pharm Sci 2019;136:104956.

24. El-Leithy ES, Makky AM, Khattab AM, Hussein DG. Nanoemulsion gel of nutraceutical co-enzyme q10 as an alternative to the conventional topical delivery system to enhance skin permeability and anti-wrinkle efficiency. Int J Pharm Pharm Sci 2017;9:207-1.

25. Vesper EO, Hammond MA, Allen MR, Wallace JM. Even with rehydration, preservation in ethanol influences the mechanical properties of bone and how bone responds to experimental manipulation. Bone 2017;97:49-53.

26. Bitto A, Burnett BP, Polito F, Levy RM, Marini H, Stefano VD, et al. Genistein aglycone reverses glucocorticoid-induced osteoporosis and increases bone breaking strength in rats: a comparative study with alendronate. $\mathrm{Br}$ Pharmacol 2009;156:1287-95.

27. Campbell GM, Sophocleous A. Quantitative analysis of bone and soft tissue by micro-computed tomography: applications to ex vivo and in vivo studies. Bone key Rep 2014;3:564.

28. Oksztulska Kolanek E, Znorko B, Michałowska M, Pawlak K. The biomechanical testing for the assessment of bone quality in an experimental model of chronic kidney disease. Nephron 2016;132:51-8.

29. Joy A, Chaitra N, Ashok M, Handral M. Antiosteoporotic activity of anthraquinone isolated from morinda citrifolia fruits in rats. Asian J Pharm Clin Res 2016;9:209-13.

30. Greenblatt MB, Tsai JN, Wein MN. Bone turnover markers in the diagnosis and monitoring of metabolic bone disease. Clin Chem 2017;63:464-74. 